\title{
Du bon usage de l'environnement par les armées.
} Le début des stratégies nationales militaires de développement durable

\author{
On the Correct Use of the Environment by the Army. \\ The Origin of National Military Strategies and Sustainable \\ Development
}

\author{
Del buen uso del medio ambiente por el ejército. \\ El comienzo de las estrategias nacionales militares en el \\ desarrollo sostenido
}

\section{Philippe Boulanger}

Volume 54, numéro 152, septembre 2010

URI : https://id.erudit.org/iderudit/045649ar

DOI : https://doi.org/10.7202/045649ar

Aller au sommaire du numéro

Éditeur(s)

Département de géographie de l'Université Laval

ISSN

0007-9766 (imprimé)

1708-8968 (numérique)

Découvrir la revue

Citer cet article

Boulanger, P. (2010). Du bon usage de l'environnement par les armées. Le début des stratégies nationales militaires de développement durable. Cahiers de géographie du Québec, 54(152), 313-336. https://doi.org/10.7202/045649ar

\section{Résumé de l'article}

De tout temps, le stratège s'est intéressé à l'environnement, non pour le protéger mais pour le malmener. Les grands théoriciens de la stratégie depuis Sun Tse, dans L'Art de la guerre, au $\mathrm{V}^{\mathrm{e}}$ siècle av. J.-C., ont montré qu'une manoeuvre se réussissait à moindre coût lorsque les données naturelles étaient exploitées avec audace. En contrepartie, les effets sur l'environnement sont peu, voire pas du tout, pris en compte. La guerre et l'environnement sont antinomiques dans nos cultures militaires occidentales. Lorsque l'armée américaine mène une guerre dévastatrice au Viêtnam, entre 1965 et 1973, elle prend bien conscience que le changement du climat ou la défoliation massive de la jungle peut servir ses intérêts tactiques contre le Viêt-cong. En revanche, elle mesure encore peu que la dégradation de l'environnement à grande échelle, relatée par les médias présents sur place et parfois par les géographes, va affecter une grande partie de l'opinion publique internationale. Il en résulte, dès 1976, une prise de conscience de la dégradation de l'environnement en temps de guerre et un début de conception commune en matière de protection dans les conflits armés, puis l'élaboration de stratégies nationales de développement durable dans les années 2000. Mais ce « bon usage de l'environnement par le militaire » reste encore limité aux armées modernes, autrement dit aux pays riches, à des fins moins militaires que politiques. Quelles sont ces stratégies environnementales nouvelles et à quoi peuvent-elles servir dans un contexte international qui prédit de grandes catastrophes écologiques dans le siècle à venir? 


\title{
Du bon usage de l'environnement par les armées. Le début des stratégies nationales militaires de développement durable
}

\author{
On the Correct Use of the Environment by \\ the Army. The Origin of National Military \\ Strategies and Sustainable Development \\ Del buen uso del medio ambiente por el \\ ejército. El comienzo de las estrategias \\ nacionales militares en el desarrollo \\ sostenido
}

\author{
Philippe BOULANGER \\ Professeur des Universités en géographie \\ PBoulanger1@gmail.comr
}

\begin{abstract}
Résumé
De tout temps, le stratège s'est intéressé à l'environnement, non pour le protéger mais pour le malmener. Les grands théoriciens de la stratégie depuis Sun Tse, dans L'Art de la guerre, au Ve siècle av. J.-C., ont montré qu'une manœuvre se réussissait à moindre coût lorsque les données naturelles étaient exploitées avec audace. En contrepartie, les effets sur l'environnement sont peu, voire pas du tout, pris en compte. La guerre et l'environnement sont antinomiques dans nos cultures militaires occidentales. Lorsque l'armée américaine mène une guerre dévastatrice au Viêtnam, entre 1965 et 1973, elle prend bien conscience que le changement du climat ou la défoliation massive de la jungle peut servir ses intérêts tactiques contre le Viêt-cong. En revanche, elle mesure encore peu que la dégradation de l'environnement à grande échelle, relatée par les médias présents sur place et parfois par les géographes, va affecter une grande partie de l'opinion publique internationale. Il en résulte, dès 1976, une prise de conscience de la dégradation de l'environnement en temps de guerre et un début de conception commune en matière de protection dans les conflits armés, puis l'élaboration de stratégies nationales de développement durable dans les années 2000. Mais ce «bon usage de l'environnement par le militaire» reste encore limité aux armées modernes, autrement dit aux pays riches, à des fins moins militaires que politiques. Quelles sont ces stratégies environnementales nouvelles et à quoi peuvent-elles servir dans un contexte international qui prédit de grandes catastrophes écologiques dans le siècle à venir?
\end{abstract}

\section{Mots-clés}

Géographie militaire, dégradation et protection de l'environnement, conflits.

\begin{abstract}
Since time immemorial, strategists have taken a keen interest in the environment, not to protect but to harm it. The major strategy theorists since Sun Tzu, who authored The Art of War in the fifth century B.C., have shown that a successful manoeuvre costs less when nature is exploited boldly. By contrast, the effect on the environment was given little consideration, if any at all.
\end{abstract}


War and the environment are diametrically opposed in Western military culture. From 1965 to 1973, when the United States army waged its destructive war in Vietnam, it realized more and more that climate change and the full-scale exfoliation of the jungle could serve its tactical plans to thwart the Vietcong. However, it underestimated the effect the extensive degradation of the environment reported by the media on site and occasionally by geographers would have on a significant part of international public opinion. Starting in 1976, an awareness of the damage to the environment in time of war and the beginning, in 2000, of a common appreciation of environmental protection as well as the development of national sustainability strategies was growing. But this "correct use of the environment by the army continues" to be restricted to modern armies - in other words, to affluent countries - not so much for military as for political reasons. So what are these new environmental strategies - and how will they be implemented in an international context where the prospect of major ecological disasters happening during the next century is looming large?

\section{Keywords}

Military geography, environmental degradation and protection, conflict.

\section{Resumen}

Desde tiempos inmemorables, el estratega se ha interesado al medio ambiente, no para protegerlo pero para maltratarlo. Los grandes teóricos de la estrategia, desde Sun Tse en El Arte de la Guerra, siglo V av. J.-C., han demostrado que una maniobra militar será un éxito y menos costosa si los elementos naturales son explotados audazmente. En cambio, los efectos sobre el medio ambiente son poco o nada considerados. En nuestras culturas militares occidentales guerra y medio ambiente son antinómicos. En la destructora guerra del Vietnam, entre 1965 y 1973, el ejército estadounidense toma conciencia que el cambio climático o la defoliación masiva de la selva puede servir a sus intereses tácticos contra el Viêt-cong. En cambio, ese ejército no comprende que la degradación del medio ambiente a gran escala, relatada por los medios de información presentes en la zona de guerra y a veces por geógrafos, va influenciar una gran parte de la opinión pública internacional. Desde 1976 surge una toma de conciencia de la degradación del medio ambiente en tiempos de guerra y un comienzo de una idea común en materia de protección en los conflictos armados y, en los años 2000, una elaboración de estrategias nacionales de desarrollo sostenido. Pero ese «buen uso del medio ambiente por los militares » se limita a los ejércitos modernos, es decir, a los países ricos, con fines más políticos que militares. ¿Cuáles son las nuevas estrategias medio-ambientales y a qué pueden servir en el contexto internacional que predice grandes catástrofes ecológicas en el siglo?

\section{Palabras claves}

Geografía militar, degradación y protección del medio ambiente, conflictos. 
a relation entre le militaire et l'environnement est d'abord à comprendre en termes d'exploitation tactique et opérationnelle. L'environnement est, dans la tradition militaire occidentale, une arme de guerre, puis un objet et un espace atteints par l'action militaire, enfin, une source de conflits.

\section{L'environnement, arme de guerre}

Le rapport entre le militaire et l'environnement renvoie traditionnellement à une notion contradictoire avec celle de développement durable. Le militaire est un acteur de la dégradation du milieu naturel à des fins tactiques dans le cadre d'une manœuvre défensive ou offensive. Dès l'Antiquité, l'empoisonnement des puits, le détournement des eaux - recommandé par le stratège chinois Sun Tse dans L'Art de la guerre (Ve siècle av. J.-C.) ou par le stratège romain Frontin dans Les stratagèmes (Ier siècle apr. J.-C.) - et le défrichement par le feu sont des moyens tactiques récurrents. Dans toutes les guerres, l'environnement devient un des moyens de se défendre ou de diminuer les capacités de l'adversaire. La provocation d'inondations comme arme de guerre en est un exemple significatif. Pendant la Grande Guerre, sur le front ouest, l'armée belge provoque des inondations dans la région de Nieuport, en Flandre, grâce au dense réseau de ruisseaux et de rivières (l'Yser notamment) ainsi qu'au relief plat, inférieur au niveau de la mer en certains endroits. Pour contrer la progression de l'armée allemande, qui atteint Dixmude en octobre 1914, le haut commandement belge décide d'inonder la rive gauche de l'Yser en fermant les écluses de Nieuport. L'eau douce cesse de couler vers la mer par les canaux (les watergrands) et inonde la wateringue (la montée continue). Les Allemands sont contraints de se retirer quelques jours après. Quelques mottes de terre restent toutefois émergées où se sont installés les avant-postes. Par ailleurs, les sapeurs belges maintiennent le niveau de l'eau à 3,30 m de sorte que, pendant quatre ans, ce secteur du front est l'un des moins agités. Il faut toutefois attendre la Seconde Guerre mondiale pour voir une nouvelle dimension dans l'emploi de l'environnement dans la guerre totale. La destruction des barrages de la Ruhr par l'aviation britannique, en 1943, est considérée comme l'une des premières utilisations militaires «modernes» de l'environnement. Cent vingt usines, 25 ponts et 25 stations d'épuration sont ainsi détruits, auxquels s'ajoutent les milliers de mines noyées rendant difficile la reconstruction immédiate. Cette opération résulte d'une manœuvre planifiée d'envergure et conduit à une catastrophe écologique majeure.

De tout temps, la destruction de certaines ressources naturelles est une cible militaire privilégiée dès les premiers jours de guerre. Durant la guerre des ports à Beyrouth en 1989 et entre les milices chrétiennes en 1990, les stations de traitement des eaux sont volontairement détruites. Cet objectif de destruction est une permanence dans les conflits contemporains depuis le $\mathrm{XX}^{\mathrm{e}}$ siècle, comme le montre la première guerre du Golfe de 1991. En janvier 1991, Saddam Hussein ordonne la destruction des sites d'exploitation pétrolière dans le but de créer une pollution marine, le dysfonctionnement des usines de traitement de l'eau salée et des obstacles à un possible débarquement de la coalition. Les forces irakiennes mettent le feu à 60 puits de pétrole le 21 janvier, puis jusqu’à 613 (sur 732 dynamités) à la fin de février, produisant une 
catastrophe écologique équivalant à 12 fois, selon le Pentagone, le désastre de l'Exxon Valdez en Alaska en 1989 (Schmitz et al., 1999). L'équivalent de 6 à 13 millions de barils de pétrole brûle chaque jour. À cela s'ajoutent les effets du nuage de fumée faisant baisser la température de 10 à $15^{\circ} \mathrm{C}$ et contaminant, par le dépôt de la suie et les pluies, $953 \mathrm{~km}^{2}$ de désert jusqu'en Turquie et en Iran. Au final, on estime que l'équivalent de 60 millions de barils se sont écoulés, formant 246 lacs pétroliers (sur une surface de $49 \mathrm{~km}^{2}$ ). Cinq pour cent de ce pétrole déversé ne peut pas être recueilli pendant les neuf mois suivant la guerre, contaminant ainsi les nappes phréatiques d'eau douce du Koweit (40\% des réserves). Bien d'autres dégâts à l'environnement sont provoqués par les armes de guerre comme les mines (500 000) posées par les Irakiens, les bombes à fragmentation (60 000), les mini-bombes (1 million lancées par les Alliés), les bombes à effet de souffle, les éclats d'obus toxiques en uranium 238 ou par d'autres effets comme les manœuvres de troupes et de blindés érodant la mince croûte qui protège le sol, le sabotage par les Irakiens au Koweit de 600 transformateurs électriques déversant des dioxines, les déchets et les eaux usées (50 millions de litres) générés par les 700000 membres de la Coalition. Selon l'Institut de recherche scientifique du Koweit, plus de $900 \mathrm{~km}^{2}$ de désert sont endommagés par les militaires, provoquant des avancées de dunes, la recrudescence de l'érosion et des tempêtes de sable (Pearce, 2000).

Il existe ainsi une permanence dans la conception de la guerre qui consiste à se servir de l'environnement comme une arme de guerre. Les doctrines militaires des armées occidentales durant la guerre froide le mentionnent clairement et définissent des procédés tactiques, sans en mesurer les conséquences environnementales, afin de créer une «contre-mobilité» et ralentir ou arrêter la manœuvre adversaire. À partir de la Seconde Guerre mondiale, la doctrine de l'armée de terre française reconnaît officiellement des pratiques déjà existantes de longue date. Elle codifie de manière théorique l'utilisation des inondations par la destruction d'un barrage amont de retenue ou des digues de protection comme l'un des six types d'obstacles, exploitant soit les données naturelles, soit des composants fabriqués, à réaliser sur le terrain (ministère de la Défense, 1980; Boulanger, 2006 et 2009). Dans le déroulement des opérations, certaines armées mettent en pratique leur doctrine où l'engagement contre l'adversaire consiste à se servir de l'environnement comme arme de guerre. En 1992, pendant la guerre de l'ex-Yougoslavie, l'armée serbe détruit sciemment le barrage situé en amont de la ville de Split (20 000 habitants), en Croatie, pour atteindre les populations civiles. Tous les moyens sont ainsi valables pour attaquer les populations civiles et les armées adverses. De fait, se distinguent plusieurs formes d'intervention du militaire sur la dégradation de l'environnement.

\section{La guerre, facteur de dégradation de l'environnement}

L'action de l'homme bouleverse l'évolution du milieu naturel. La dégradation de l'écosystème par la mise en valeur agricole des terres, l'essor des activités industrielles et l'urbanisation est un fait dénoncé par l'opinion publique internationale. La guerre fait partie de ces facteurs qui touchent directement le milieu naturel. Nombreux sont les exemples de destruction du milieu naturel jusqu'alors préservé. En France, les forêts lorraines, entre autres celles de l'Argonne ou de Verdun, subissent encore, un siècle après la Première Guerre mondiale, les séquelles des bombardements d'artillerie. 
Les préoccupations des militaires ont été longtemps éloignées de la protection de l'environnement. L'influence du militaire apparaît au moins à quatre niveaux provoquant de profonds changements sur l'écosystème.

Le premier niveau porte sur les défrichements. La création de bases militaires conçues comme de véritables villes, de terrains d'aviation et de moyens de transport (routes, voies ferrées, canaux), ainsi que l'emploi de différents systèmes d'armes conduisent à modifier le cadre environnemental. L'impact de ces aménagements sur l'écosystème, le plus souvent difficilement mesurable à défaut d'études scientifiques, peut être irréversible. La construction de lignes fortifiées et de fortifications bouleversent le milieu naturel. Le Mur de l'Atlantique, édifié par l'organisation Todt entre 1941 et 1944, des côtes de Norvège à la frontière franco-espagnole, laisse des traces dans l'environnement encore visibles aujourd'hui. Les constructions en béton, murs et bunkers, accélèrent l'action de l'érosion marine sur le littoral.

Le deuxième niveau concerne les bombardements et la défoliation. Les munitions classiques sont source de dégradation de l'environnement. Au stade de la fabrication, les matières premières (plomb et autres métaux lourds) de même que les procédés utilisés, comme les solvants pour la synthèse des matériaux énergétiques, présentent des risques d'impact environnemental. Au stade de l'utilisation opérationnelle, les poudres et les propulseurs dégagent des produits de combustion et des résidus, des produits de détonation ainsi que des oxydes de métaux lourds. La pollution du champ de bataille se maintient par les corps de munition et la substance active non explosée. Lors du retrait du service de la munition, se pose le problème de son retraitement. Les munitions sont, avec les carburants, les plus importants éléments à risque environnemental dans les systèmes d'armes. Dans la phase d'emploi, la disparition d'espèces végétales et animales est l'une des conséquences majeures. Ce phénomène se produit surtout lorsqu'une ligne de front est établie. Durant la guerre américaine du Viêtnam (1965-1972), une tactique de destruction massive de l'écosystème par raids aériens, visant les armées viêt-cong, est mise au point dès 1967. Toutes les concentrations d'armées vietnamiennes sont visées par les B-52, volant par groupe de trois. Chaque bombardier lâche au minimum 40 bombes de 500 livres à $9000 \mathrm{~m}$ d'altitude, de jour comme de nuit, par n’importe quel temps. La précision des bombardements est fixée selon un système de coordonnées exactes de radio-balises fixes enregistrées sur ordinateur. Des zones entières sont ainsi ravagées. Les dégâts à l'environnement, comme les pertes humaines, sont considérables.

$\mathrm{Au}$ cours du XXe siècle, d'autres agents de destruction durable sont entrés en action. Durant la guerre américaine du Viêtnam, pour déstabiliser la tactique de guérilla des Viêt-cong dissimulés dans la jungle, les militaires américains ont fréquemment recours aux défoliants comme l'Agent Orange (résidu de dioxine à effet durable pendant trois à cinq ans). Ceux-ci sont employés dès 1962 par l'armée de l'air américaine avec l'autorisation de la République du ViêtNam et leur emploi donne des résultats limités. Mais les zones touchées, surtout dans la péninsule de Cau Mau dans le delta du Mekong, sont devenues hautement polluées et, pour certaines, démilitarisées. On estime que deux millions d'hectares de forêt sont détruits à la suite de leur déversement. D’autres agents chimiques sont aussi employés, comme le napalm plus efficace que les explosifs de forte puissance, accélérant le processus d'érosion et favorisant les inondations. Les sols sont devenus hydromorphiques et impropres à l'agriculture. Des 
espèces végétatives ne peuvent repousser en raison du sol contaminé conduisant à l'apparition de nouvelles espèces de faune et de flore. Les conséquences écologiques sont estimées irréversibles et ont profondément modifié non seulement l'écosystème, mais aussi la vie des sociétés rurales.

Le troisième niveau d'influence renvoie à la maîtrise des conditions climatiques et à leur détournement à des fins militaires. Après la Seconde Guerre mondiale, de nombreuses expériences sont programmées pour parvenir à provoquer des climats favorisant une opération militaire. En 1946, des ingénieurs de l'entreprise General Electric découvrent par hasard que des particules de glace sèche (dioxde de carbone gelé), déposées dans une chambre froide, forment des cristaux de glace similaires à ceux présents dans les nuages. Cette découverte effectuée par des civils est à l'origine des programmes pour provoquer des pluies dans les régions touchées par la sécheresse. Par ailleurs, elle intéresse aussi les militaires. La première modification volontaire du climat par les militaires apparaît durant la guerre du Viêtnam au cours de l'opération Popeye (Durschmied, 2004). À partir de la base aérienne d'Udorn en Thaïlande, trois WC-130 du Service météorologique de l'armée de l'air américaine larguent des fusées d'iodure d'argent et de plomb pour créer des nuages. Ils opèrent à la fois au Nord-Viêtnam, au Cambodge, au Sud-Viêtnam et au Laos en effectuant 12000 sorties entre 1967 et 1972. Le but consiste à prolonger la mousson et à provoquer des pluies endommageant la piste Hô-Chi-Minh empruntée par les armées nord-vietnamiennes pour ravitailler leurs partisans au sud du pays. Cette modification du climat, révélée à l’opinion publique au début des années 1970, provoque de telles protestations qu'elle doit être arrêtée. À cela, s'ajoute le fait que les effets sur l'adversaire se montrent peu rentables. Si l'opération Popeye se révèle un échec sur le plan tactique, en revanche, le largage de sel à partir d'avion cargo pour faire disparaître le brouillard matinal sur les pistes des bases aériennes, notamment celle de Khe Sanh assiégée en 1968, est un succès. Le procédé est d'ailleurs exploité depuis pour assurer le fonctionnement des aéroports civils.

La maîtrise du climat constitue toujours un enjeu essentiel pour le militaire, bien que des mesures internationales en interdisent la pratique. À la suite des révélations faites sur l'opération Popeye, des négociations entre les États-Unis et l'URSS débutent dès juillet 1974 pour prévenir les tentatives de modification de l'environnement. Elles conduisent à la résolution des Nations Unies du 3 septembre 1976 interdisant de modifier le climat en temps de guerre. Cette résolution est signée à Genève le 18 mai 1977 par 25 pays dans le but d'améliorer l'environnement et son utilisation à des fins pacifiques. Cela n'empêche cependant pas plusieurs armées de lancer des programmes de recherche sur la maîtrise du climat pour le temps de guerre. En 1994, par exemple, le plan USAF Spacecast 2000 prévoit de modifier le climat pour atteindre l'adversaire. En 2002, l'US Air War College présente une étude intitulée «Maîtriser le climat en 2025 ». La maîtrise du climat ne cesse de représenter un enjeu essentiel pour le militaire.

Enfin, le quatrième niveau regroupe tous les nouveaux risques (nucléaire, bactériologique et chimique) apparus au $\mathrm{XX}^{\mathrm{e}}$ siècle. Certains sites peuvent être menacés en devenant les cibles d'une guerre future. Les centrales nucléaires (pollution par les éléments radioactifs), les sites chimiques, gaziers, pétroliers sont des armes de guerre pouvant mettre en péril l'équilibre de l'environnement et des sociétés en cas d'attaque déclarée ou terroriste. Déjà, en juin 1981, l'aviation iranienne s'est attaquée 
au réacteur de Tuwaitha en Irak. Le risque de catastrophe écologique apparaît donc prépondérant, notamment concernant les centrales nucléaires dont 429 sont en activité dans le monde ${ }^{1}$. Dans les armées occidentales, le militaire en opérations doit tenir compte du risque biologique naturel, source de stress, ainsi que des risques chimiques et radiologiques dans l'environnement industriel. Des mesures de précaution selon les types d'engagement sont ainsi prises. Dans l'armée française, on a mis en place la chaîne Hygiène et sécurité des militaires en opérations, qui témoigne de la prise de conscience face au danger biologique et chimique, en vue de la décontamination d'une zone ${ }^{2}$. De plus en plus, la préparation et la mise en œuvre des mesures de prévention sont intégrées dans la planification opérationnelle ${ }^{3}$.

La guerre est donc un facteur de dégradation de l'environnement qui s'accentue avec l'emploi potentiel d'armes chimiques et nucléaires. Dès les années 1970, certains géographes se sont publiquement manifestés contre la croissance de ce phénomène. En juin 1972, le géographe Yves Lacoste dénonce, dans Le Monde, la stratégie de destruction par bombardement aérien des digues du delta du Fleuve rouge, survenu au Nord-Viêtnam entre mai et juillet 1972. Il montre ainsi que les points bombardés visent les zones sensibles des digues: la partie la plus basse du delta où les bras du fleuve, qui surplombent de nombreux villages, sont les plus nombreux, la partie concave des méandres (la plus fragile en raison de la pression du courant), la partie en contrebas de la digue pour faire céder la paroi (et non frontalement, ce qui aurait été dénoncé par les médias et la propagande). Cette stratégie conçue par l'armée américaine doit avoir pour résultat, lors de la prochaine saison des pluies, d’inonder le delta et de créer une catastrophe écologique pénalisant toute l'organisation des sociétés rurales et la résistance nord-vietnamienne. L'argumentation d'Yves Lacoste, illustrée par plusieurs cartes et reprise par la presse internationale, aurait influencé l'opinion publique internationale, y compris le pape Paul VI qui a fait part de ses inquiétudes au président américain Nixon (Lacoste, 2008).

L'environnement utilisé comme une arme de guerre commence à susciter plus d'intérêt de la part de la communauté scientifique à partir de cette époque. Apparaît également une autre dimension qui suscite plus d'intérêt et qui s'inscrit comme une cause ou une conséquence de la précédente.

1 En France, par exemple, ces différents sites font l'objet d'une surveillance et d'une défense militaire (batterie sol-air contre une attaque suicide aérienne) renforcée depuis les attentats du 11 septembre 2001 à New York.

2 Cette prise en compte du risque environnemental sur le terrain repose sur une procédure en quatre étapes. La première est celle de l'anticipation par la recherche du renseignement sur l'environnement. On constitue ainsi des bases de données à partir des sources civiles et militaires. Pour répondre à ces objectifs, est créé le pôle technique de la Cellule d'analyse des risques biologiques et chimiques de l'Etat-major de l'armée adossé à l’Institut de médecine tropicale du Service de santé des armées. La deuxième étape est celle de l'analyse et de l'évaluation de l'intensité du risque. Elle vise à détecter et à identifier la contamination NRBC (nucléaire, radiologique, biologique et chimique) dont le risque est redouté par les troupes en campagne. La troisième étape est celle de la définition du seuil d'exposition tolérable à partir des références rassemblées par des experts auprès du commandement. Enfin, la dernière phase est celle de l'application des mesures préventives et de la gestion des incidents. (Marionnet, 2003: 41-44).

3 À ce stade, le militaire se heurte cependant à deux problèmes : celui de la connaissance imparfaite de l'environnement des opérations et celui des investigations limitées. Pour limiter les effets inattendus, sont aussi élaborées des parades contre les risques comme la fabrication de matériaux et de revêtements ne fixant pas les toxiques. Parallèlement, des études de détecteurs à spectre plus étendu (matériaux lourds, toxiques chimiques différenciés) sont réalisées. 


\section{La maîtrise de l'environnement, source de tensions}

Ainsi, la dégradation de l'environnement peut provoquer de nouvelles sources de tension sous la forme d'un «conflit vert». Selon l'Institut de recherche et d'information sur la paix et la sécurité, cette notion recouvre «des situations explosives qui peuvent aller de l'émeute locale jusqu'au conflit international en passant par l'insurrection, la répression ou la guerre civile et qui ont comme particularité d'être directement liées au changement de l'environnement» (Sironneau, 2003 : 100).

Trois types de conflit peuvent émerger en rapport avec le facteur environnemental. Le premier est lié à la pénurie des ressources environnementales, comme l'eau dont la maîtrise peut être le prétexte au déclenchement d'un conflit aux causes plus étendues. En Somalie, le chaos politique qui s'est instauré depuis 1991 s'explique par diverses raisons. Le pays sombre dans une guerre civile qui conduit à l'indépendance - non reconnue par la communauté internationale - de plusieurs entités territoriales comme le Somaliland au Nord. Cette région semi-aride de savane et de steppe, à l'exception du sud bénéficiant des grands fleuves et de cultures irriguées, souffre d'une pénurie d'eau. Les pluies se sont raréfiées, les nappes d'eau souterraines s'amenuisent, les puits ferment, obligeant les populations nomades (70\% de la population totale) à se sédentariser dans les villes. Quand l'eau est accessible, elle apparaît contaminée par des pesticides interdits en Occident. Il en résulte une tension croissante concernant l'accès aux ressources naturelles disponibles et des affrontements pour le contrôle des puits. Les troupeaux (six millions de têtes au début des années 2000) se concentrent dans certaines zones. Cette concentration accélère encore l'épuisement des ressources en eau, ainsi que l'érosion des sols liée au surpâturage et à la disparition des arbres. Vadrot (2005) considère ainsi que $100 \mathrm{~km}^{2}$ de zone boisée disparaissent chaque mois.

Le deuxième type est lié à la raréfaction de la ressource environnementale et à l'identité du groupe (ethnique, religieux nationaliste). Depuis les années 1990, l'Afrique des Grands Lacs (Ouganda, Burundi, Rwanda, Tanzanie, République démocratique du Congo) traverse de multiples crises de violence (génocide, guerres régionales étatiques, guerres interethniques et intraétatiques) liées à la gestion et à l'exploitation des ressources naturelles. Les forêts tropicales sont intensément défrichées par des sociétés internationales. Le bois précieux, recherché en Occident et en Asie, est échangé contre des armes pour les milices locales. Il est transporté en avion par l'Ouganda ou le Kenya, celui moins précieux par Kinshasa (RDC) et le port de Borna sur le fleuve Congo. Il en résulte plusieurs conséquences. Outre la disparition progressive d'espèces animales, comme le singe Bossolo en RDC commercialisé pour sa viande, il se produit une désertification des forêts de la région. On estime que $600 \mathrm{~km}^{2}$ de la forêt de Gishwati près de Kigali et $400 \mathrm{~km}^{2}$ de celle du parc naturel d'Akagira ont été rasés. À la suite des conflits entre ethnies, les parcs nationaux, comme ceux du Kivu dans l'est de la RDC, sont devenus des zones refuges pour les populations déplacées et la base arrière des groupes armés congolais, rwandais et soudanais. 
Le troisième type de conflit vert est lié à la discrimination et à la privation d'une ressource environnementale ${ }^{4}$. Dans l'est de la Turquie, la population kurde est ainsi l'objet d'une politique discriminatoire menée par le gouvernement d'Ankara afin de réduire les capacités de ses groupes armés (PKK) depuis la fin des années 1970. La construction de grands barrages bouleverse l'équilibre environnemental. Outre la destruction de plus de 4000 villages, entre 500000 et 1,5 million de personnes doivent quitter les zones sans ressources en eau et sont amenées à se déplacer des campagnes vers les villes où leur contrôle se révèle plus facile. Dans bien d'autres régions, l'exploitation des ressources naturelles conduit à priver les populations locales de leur identité et de leur mode de vie ancestral. En Malaisie, à la fin des années 1980, les populations de Sarawak se heurtent à la politique du gouvernement qui procède à l'abattage de la forêt (Schmitz et al., 1999). Dans le nord-ouest de l'Amazonie, les Yanomani sont envahis par les sociétés d'exploitation des ressources locales comme l'or, le diamant, l'uranium, le titane et l'étain. L'exploitation de la ressource halieutique peut aussi conduire à des conflits. Entre pêcheurs traditionnels et gros chalutiers, des accrochages au sujet des quotas de pêche et de la maîtrise des zones de pêche peuvent éclater, comme au large des côtes mauritaniennes avec la flotte russe ou dans les Caraïbes avec la flotte américaine. Dans bien d'autres régions du monde, au Darfour, au Sri Lanka, au Népal ou au Haut-Karabagh, dans les années 1990 et 2000, de semblables politiques de privation de ressources naturelles, liées à l'eau, aux terres arables, à la pêche et au bois principalement, sont adoptées directement ou indirectement pour soumettre et contrôler des groupes ethniques.

Ces différentes formes de conflits liés à l'environnement accroissent les flux de réfugiés ruraux vers les villes, accélérant ainsi le processus de périurbanisation et d'urbanisation des espaces ruraux. La raréfaction de la ressource en eau aurait provoqué l'exode rural de 25 millions de personnes dans le monde à la fin du XXe siècle (Sironneau, 2003). Pour la construction du barrage des Trois-Gorges en Chine, 10 millions de personnes sont ou seront déplacées des campagnes vers les villes ${ }^{5}$. En Inde, 33 millions de personnes auraient été contraintes de laisser leurs campagnes pour la construction des 4600 barrages dont 1600 sont en cours d'érection. Ces populations «écoréfugiées» deviennent également des sources de déstabilisation d'un État ou d'une région. Elles participent aux déséquilibres des structures politiques existantes, souvent fragiles, et à la formation des «zones grises» comme en Afrique de l'Est (Somalie au début des années 1990) et en Amérique du Sud (Colombie, Pérou, Bolivie). Les modifications environnementales provoquées par les politiques d'aménagement du territoire, nationales ou régionales, portent les germes de nouvelles tensions (Indonésie à propos

4 Ce type de situation est représenté par la politique de restriction et d'autorisation israélienne de la consommation de l'eau en Cisjordanie. En se voyant interdits les forages et en étant soumis à un système de quotas, les Palestiniens sont d'une manière indirecte incités à quitter ces territoires. Au Brésil, l'évolution des fronts pionniers dans la forêt amazonienne conduit au déplacement des populations indiennes privées de leurs ressources premières reposant sur le bois. On estime que 50000 Indiens sont ainsi déplacés lors de la mise en eau des barrages d’Itaipu et de Tucurui.

5 Ce chantier a commencé dans les années 1990 et a été achevé en 2008. La construction du barrage des Trois-Gorges dompte les eaux du Chang Jiang. Grâce à cet ouvrage colossal, les navires de haute mer peuvent remonter le fleuve jusqu'à Yichang, à $1500 \mathrm{~km}$ de la mer. Sa puissance électrique de 18200 MW devrait alimenter la population dans un rayon de $1000 \mathrm{~km}$. En contrepartie, 300 villages ont été engloutis et un million de personnes ont été déplacées dans le court terme. 
de la gestion de la forêt), de guerre civile (Rwanda-Burundi sur le contrôle de la terre arable) ou interétatique (gestion de l'eau au Proche-Orient et Moyen-Orient) qui s'ajoutent généralement à d'autres, d'ordre politique, économique, culturel et social.

En somme, dans un premier temps, la notion d'environnement apparaît fortement éloignée de celle de protection ou de développement durable. Pourtant, elle commence, à partir des années 1970, à préoccuper le militaire, principalement dans les armées occidentales. Le risque environnemental de la guerre est pris en compte en temps de paix. On tend à mieux appréhender les effets des systèmes d'armes et à intégrer le facteur environnemental dans les diverses opérations militaires.

\section{Du bon usage de l'environnement dans les armées}

Depuis la guerre américaine du Viêtnam, une nouvelle conception de l'environnement se dégage dans les mentalités. D’arme de guerre qu'il était, l'environnement fait graduellement l'objet de politiques de protection, d'abord dans les armées des États neutres, puis aux États-Unis, en Angleterre et en France. Quel est cet usage nouveau de l'environnement par les armées?

\section{La Convention sur l'emploi militaire de l'environnement de 1976: une rupture culturelle et juridique majeure}

Pendant la guerre américaine du Viêtnam, qui s'achève en 1973, l'opinion publique internationale s'émeut de la dégradation de l'environnement naturel par l'emploi de tactiques spécifiques et de nouvelles armes aux effets durables. On critique les méthodes de destruction des forêts et des récoltes comme les tentatives de manipulation du climat par l'armée américaine. En réaction, une conférence d'urgence contre la guerre environnementale se réunit en juin 1972 à Stockholm. Devant la multiplication des dénonciations, la communauté internationale décide d'adopter une convention qui définirait les limites de l'utilisation des conditions environnementales dans les conflits futurs. La première base de ce processus, en cours dans les années 1970, est la Convention ENMOD du 10 décembre 1976. Parallèlement, d'autres déclarations aboutissent au même constat. Le rapport du sénateur Clairborne Pell au Sénat américain de juillet 1973, le projet suédois à la conférence du Comité de désarmement pour la mise au point de méthodes de guerre météorologique et la déclaration américano-soviétique sur les dangers de modification de l'environnement du 3 juillet 1974 expriment des intentions identiques. La plus importante est la proposition conjointe (États-Unis-URSS), adoptée lors de la conférence sur le désarmement le 21 août 1975, en faveur d'une interdiction des techniques modifiant l'environnement à des fins militaires avec des effets durables. Les conditions de mise au point d'un texte définitif ne sont cependant pas précisées.

La dégradation de l'environnement comme arme de guerre y est théoriquement interdite. Décidée par l'Assemblée générale des Nations Unies et entrée en vigueur le 5 octobre 1978, la convention qui résulte de cette proposition est ratifiée par 64 États. Quinze autres la signent sans la ratifier, et neuf joignent des réserves à leur signature. Le document interdit l'utilisation « des techniques de modification de l'environnement à des fins militaires ou toutes autres fins hostiles». Mais cette intention demeure 
subjective dans son application. En témoigne la destruction des puits de pétrole du Koweit par l'armée irakienne pour protéger ses mouvements de retraite et entraver la manœuvre des Coalisés, en 1991. Sur un plan juridique, il est effectivement reconnu que chaque protagoniste peut modifier l'environnement à des fins militaires contre un adversaire à condition que les effets ne soient pas étendus. La convention ne vise pas non plus à réglementer les effets de la guerre, c'est-à-dire les dommages indirects de guerre. En outre, le terme «technique» conduit à des débats empreints de controverse. Si la provocation de tremblements de terre ou de tsunamis et la modification des conditions climatiques ne font pas l'objet d'interprétations spécifiques, il en va différemment des procédés de dérivation de fleuves, de terres brûlées, de la pollution volontaire des mers, des incendies de puits de pétrole et de l'emploi d'herbicides. Enfin, les États-Unis n'ont pas tendance à interdire, de façon à éviter des litiges nouveaux. On retient donc finalement que seraient interdits les «dommages étendus, durables ou graves", laissant ainsi une interprétation flexible de la convention.

Une nouvelle disposition est adoptée pour compléter la première. En 1977, les articles 35-3 et 55 du protocole I additionnel aux Conventions de Genève, relatif à la protection des victimes des conflits armés internationaux, soulignent l'intention de protéger les individus comme l'environnement (Mollard-Bannelier, 2001). Le protocole interdit l'utilisation «des méthodes ou moyens de guerre dont on peut s'attendre qu'ils causent des dommages étendus, durable et graves à l'environnement naturel» dans la mesure où la santé ou la survie des populations est compromise (Sironneau, 2003: 107). Il précise la protection des ouvrages ou des installations contenant des «forces dangereuses» comme les barrages et les digues qui peuvent être des objectifs stratégiques. En somme, les critères sont plus exigeants que ceux de la Convention EMMOD. Les articles visent à protéger l'environnement quelle que soit l'origine du dommage. Ils reconnaissent que ce dommage résulte d'une utilisation de l'environnement ou de toute autre méthode ou moyen de guerre. Le critère de durabilité est donc plus exigeant. En 2001, 152 États acceptent cette nouvelle juridiction internationale (contre 102 en 1991). Seule une trentaine ne la suivent pas, notamment Israël, le Pakistan, le Soudan, la Syrie et la Turquie. Malgré tout, on peut souligner des limites. Le champ d'application ne concerne que le territoire de l'État ennemi. Il ne précise pas la responsabilité d'un État qui détruit son propre environnement à des fins défensives. Il reste également vague concernant la zone maritime puisque le règlement n'aborde que le cas de la guerre maritime si les objectifs sont situés sur terre. Enfin, les armes de destruction massive ne sont pas mentionnées.

Bien que ces deux nouveaux règlements internationaux s’ajoutent à ceux relatifs au droit de la guerre, notamment à la protection des biens dont le règlement est annexé à la Convention de La Haye de 1907 et à la protection des personnes civiles en temps de guerre ( $4^{\mathrm{e}}$ Convention de Genève de 1949), ils présentent la nouveauté de s'intéresser uniquement à la protection de l'environnement. Reste toutefois posée la question de leur application. Jusqu'à la création de la Cour pénale internationale, en juillet 1998, il n'existe pas de compétence universelle spécifique, en dehors de la Cour internationale de justice, pour poursuivre et juger les auteurs de crimes environnementaux en temps de conflit armé. Depuis 1998, trois autorités sont habilitées à saisir la Cour de dérogations au droit international, à savoir la Cour internationale, le Procureur par un État partie et le Conseil de sécurité de l'ONU. Parallèlement à cette nouvelle juridiction, au niveau national, certaines armées commencent une révolution culturelle en adoptant des dispositions en faveur d'une "stratégie de développement durable». 


\section{La naissance de stratégies de développement durable dans les armées modernes}

À partir des années 1990, la plupart des grandes armées modernes commencent à adopter des politiques de développement durable, respectueuses de l'environnement. Pour la première fois, il semble que la notion de protection de l'environnement prédomine sur l'utilisation de l'environnement à des fins tactiques et opérationnelles. Protéger l'environnement est érigé en cause d’intérêt général dans la gestion des armées en temps de paix.

Cette prise de conscience semble apparaître d'abord au sein des armées des États neutres en Suisse, au Canada ou en Suède. La protection de l'environnement y est une approche culturelle des activités militaires en temps de paix, comme la bonne gestion des domaines militaires ou le contrôle de la pollution des sols. Au Canada, le premier plan de développement durable du ministère de la Défense, adopté en 1997 et mis à jour tous les trois ans, prévoit une démarche environnementale relative aux sites suivant les prescriptions de la norme ISO 14001. Un sous-ministère Infrastructure et environnement doté d'une direction générale Environnement traite de quatre aspects majeurs : les écosystèmes, la prévention de la pollution de l'air, le changement climatique et une gestion qui favorise le développement durable. La priorité est donnée à la formation du personnel, aux impacts environnementaux et aux outils de mesure de l'impact. Depuis 2003, les contraintes environnementales dans la conception et le processus d'acquisition des nouveaux systèmes d'armes sont mieux prises en compte. En Suède, une telle prise de conscience est également mise en œuvre. Chaque entité prépare un plan de gestion environnementale. Chaque programme fait l'objet d'une évaluation environnementale. En Allemagne, la gestion environnementale est confiée à des centres techniques spécialisés. En matière de conception d'un programme d'armement, des normes doivent être respectées: le recyclage et le traitement des matériaux sont également pris en compte. En Suisse, l’armée suit également une démarche d'écoconception en mesurant l'impact des niveaux de pollution (sonore, air) et la consommation d'énergie. La Défense mesure les bilans écologiques et effectue des analyses environnementales.

Dans les armées des grandes puissances, cette prise de conscience s'exprime ouvertement dans les années 1990 et prend une importance croissante dans les années 2000. Aux États-Unis, la protection de l'environnement s'est accentuée sous la présidence de Bill Clinton. En 1990, est créé l'Army Environmental Policy Institute dont la mission est de définir des réglementations relatives à la gestion de l'environnement au ministère de la Défense. Dès 1992, ce ministère doit rendre des comptes au Congrès et est pénalement responsable de toutes les infractions constatées. La priorité consiste à sensibiliser les militaires aux questions environnementales, à réhabiliter les sols et à prévenir les pollutions éventuelles. Par la directive 13148 Greening the goverment through leadership in environmental management, adoptée en 1997, suivie de nouvelles dispositions gouvernementales, des efforts importants sont effectués pour prévenir les pollutions et introduire l'écoconception dans les programmes d'armement (Guével, 2003 ; Chaix, 2006). La réglementation est particulièrement étendue, comprenant 40 lois et de nombreuses dispositions fédérales, ainsi que des lois locales qui imposent une transparence sur ces questions. En avril 2002, l'armée américaine adopte le programme Environmental management system qui impose aux responsables militaires 
d'établissements, pour 2009, de bâtir une politique de protection de l'environnement, d'en superviser le déroulement, d'analyser les résultats et d'améliorer le système. En 2005, l'Army Environmental Policy Institute adopte l'US Army Environmental Strategy qui définit une stratégie de développement durable («Maintenir la mission, sécuriser le futur») à long terme, y compris dans les opérations militaires. Cette stratégie prévoit réduire graduellement l'intensité énergétique de la Défense de 3\% à 30\% avant 2015. Elle lui impose aussi de diversifier ses sources d'énergies. Pour tout achat annuel de crédit d'énergie renouvelable, le ministère de la Défense doit s'assurer que la moitié de ces crédits proviennent de nouvelles sources d'énergie renouvelable. Dans le domaine des transports, il est prévu d'augmenter la part annuelle de carburant alternatif de $10 \%$.

Les armées - l'Air Force, la Navy, le Marine corps - disposent de centres de compétences spécialisés comme le Center for Environmental Excellence (400 personnes, Air Force) et l'Army environmental center (250 personnes, Army) à Aberdeen qui mène des expériences et aide les décideurs à évaluer les conséquences environnementales des programmes terrestres. Pour les trois armées, un service commun (Defense Logistics Agency) est chargé de la gestion des déchets de matériaux à risque (130 000 tonnes par an) pour un coût de 60 millions \$ par an. Il valorise les produits verts comme les soudures sans plomb, favorise les progrès en matière de biocarburants et gère les déchets électriques et électroniques ainsi que la récupération des métaux nobles. Le programme de véhicule amphibie du Marine corps, portant sur 1000 véhicules, constitue un exemple significatif en termes d'écoconception. Les matériaux à risque sont remplacés ou réduits : suppression du cadmium et de substances pouvant endommager la couche d'ozone, peinture extérieure non polluante, utilisation de polypropylène comme liquide de refroidissement à la place de l'éthylène plus difficile à détruire, produits de nettoyage biodégradables. Le Pentagone a adopté, en 2003, un protocole visant à nettoyer les installations polluées par des munitions. Sous l'égide des services du sous-secrétaire adjoint pour les installations et l'environnement, un plan d'action concerne 1691 anciens sites militaires, 542 installations opérationnelles et 74 bases en cours de fermeture pour un coût de 11,5 milliards $\$$. Il prévoit évaluer séparément le danger représenté par les risques d'explosion des munitions, les risques de pollution chimique et les risques pour la santé et l'environnement. Huit niveaux de priorité sont ainsi définis pour déterminer une échelle des risques. Ce plan d'action devrait être appliqué pendant une dizaine d'années. Des moyens importants sont donc investis pour la protection de l'environnement.

En Grande-Bretagne, les équipes de la Defense Procurement Agency n’appliquent pas de programmes spécifiques, dans les années 1990, quant à l'utilisation de matériaux et substances dangereuses pour les programmes d'armement. Le ministère de la Défense s'est cependant doté de defense standards pour traiter les aspects environnementaux. Ces documents précisent une liste de produits ou de matériaux nocifs à remplacer ou à réduire. Ils tendent à mettre en application les mesures de l'Environmental Protection Act de 1990 et de l'Environment Act de 1995 ainsi que d'autres textes internationaux. En 1998, est ainsi formée la direction Directorate of Safety Environment and Fire Policy au sein du ministère de la Défense. Certaines sociétés commencent également à mener des politiques environnementales dans la fabrication d'engins. Ainsi, en 1999, la société Rolls-Royce publie un rapport sur le sujet. Une prise de conscience de ces problèmes tend donc à se former depuis les années 1990. Toutefois, à partir des années 2000, cette réglementation s'est précisée en suivant les modèles occidentaux. 
En 2001, une convention est signée entre le Ministère et l'Agence de l'environnement britannique. En juin 2002, le Ministère adopte un plan d'action de développement durable applicable avant tout aux transports. En 2004, est créé un système de gestion environnementale, au sein de la Defense Procurement Agency, qui mène des études sur les risques dans la conduite des programmes militaires.

En France, les premières mesures datent des années 1930. La loi du 2 mai 1930, relative à la protection des monuments naturels et des sites à caractère artistique, historique, scientifique, légendaire ou pittoresque, permet d'appliquer deux dispositifs: l'inscription et le classement des sites. Cette mesure, dont la vocation est de préserver des espaces en raison de leur caractère exceptionnel, concerne peu de terrains affectés au ministère de la Guerre, mais le contenu est aujourd'hui codifié aux articles 341-1 et suivants du Code de l'environnement (Gandubert, 2003). Le ministère de la Défense est affectataire du deuxième patrimoine foncier (265000 ha) dont la moitié des terrains sont utilisés dans un cadre de manœuvres et d'exercices et n'ont jamais fait l'objet d'aménagement urbain, industriel ou agricole. La prise de conscience de la nécessité de préserver la qualité de l'environnement apparaît dès les années 1970, mais fait l'objet de mesures codifiées seulement depuis les années 1990. En 1995, on recense plus de 100000 ha de terrains de l'armée de terre française faisant l'objet de mesures de protection de la nature intégrant les inventaires (zones naturelles d'intérêt faunistique et floristique dans les années 1980) et les outils légaux (réserves naturelles, parcs nationaux, etc.). Les établissements d'essais, de stockage de carburants ou de munitions, l'entretien des chars, des aéronefs et des navires sont l'objet d'une attention environnementale plus précise. En 2006, quelque 8600 sites sont classés à risques ou susceptibles d'avoir un impact sur l'environnement ou les ressources en eau. Les écosystèmes aquatiques font l'objet de mesures spécifiques. En particulier, selon le ministère de la Défense, 1500 sites sont soumis à une réglementation sur l'eau et 2000 sites soumis à autorisation en raison de leur caractère dangereux pour l'environnement.

Les risques de pollution liés au fonctionnement de ces installations classées sont les mêmes que ceux présentés par les industries du secteur civil. Des inspections pour la protection de l'environnement sont prévues dès 1976 avec mission d'assurer la sécurité maximale des dépôts de munitions, la réglementation des transports de munitions, la sécurité des dépôts d'hydrocarbures et la réglementation de l'eau ${ }^{6}$. Ces inspections sont en dialogue permanent avec le ministère de l'Écologie et du Développement durable. Les mêmes procédures de concertation (enquêtes publiques, consultation des maires, des conseils municipaux, des conseils départementaux d'hygiène, etc.) que dans le domaine civil sont suivies à l'exception des installations couvertes par la confidentialité. Une prise de conscience réelle de la question environnementale, du développement durable et de la protection de la nature s'est donc concrétisée voici quelques années.

Avec le ministère de l’Écologie, celui de la Défense adopte en juin 2003 une charte de l'environnement, prolongeant le protocole de 1995 dont les normes s'imposent au législateur et aux autorités administratives. Cette charte, qui constitue la base de la stratégie de développement durable, vise à valoriser, à maintenir et à réhabiliter les

6 Inspection des installations classées propres au ministère de la Défense, inspections interarmées et indépendantes de la hiérarchie militaire. 
sites militaires, les espaces de manœuvres protégés, à établir des échanges fonciers avec l'Office national des forêts ainsi que des conventions avec le Conservatoire du littoral et des rivages lacustres. Composée de 10 articles, elle prévoit des actions dans le cadre de Natura 2000, un programme de prévention des pollutions et des risques (installations classées, fonds dédié à la dépollution des sols entre autres), l'amélioration de la formation du personnel civil et militaire, l'intégration du concept de développement durable (labellisation ISO 14001 des établissements et centres d'essai de la DGA, intégration de l'écoconception), la répression des atteintes à l'environnement (création de l'Office central de lutte contre les atteintes à l'environnement et à la santé publique). Pour mettre en œuvre ce protocole, cinq commissions mixtes locales sont créées dans chaque région Terre (Bordeaux, Lyon, Metz, Rennes, Saint-Germainen-Laye) et réunissent des représentants régionaux de la Défense et de la direction régionale de l'environnement. L'aliénation des sites remarquables constitue l'une des orientations importantes de cette charte car les camps militaires, les bases aériennes et les sites littoraux sont des réserves écologiques recherchées par les protecteurs de l'environnement. L'objectif est de maintenir cette qualité de l'environnement sans sanctuariser les sites avec le risque d'interdire toute activité.

Dans le prolongement de ce protocole, la Défense signe en décembre 2006 une convention d'une durée de 10 ans avec le Conservatoire de l'espace littoral et des rivages lacustres (CELRL). Le Ministère s'engage à céder au CELRL (ou à établir avec lui des conventions de gestion) une trentaine de sites représentant 1500 ha, afin de contribuer à leur sauvegarde écologique. Avec l'Office national de la chasse et de la faune sauvage, une autre convention est signée le même mois concernant la conservation et la gestion de la faune sauvage sur les domaines militaires ( 25 sites pilotes) présentant un intérêt faunistique remarquable. Enfin, avec l'Office national des forêts (ONF), le Ministère confie la gestion d'une partie de son domaine boisé dans le cadre de conventions locales. L’ONF assure l'entretien, la régénération et éventuellement des travaux de reboisement des terrains militaires.

Plus récemment, à la suite du Grenelle de l'Environnement, le ministère de la Défense a prolongé le protocole de 2003. En décembre 2007, est lancé le plan d'action Environnement qui prévoit 40 mesures en matière d'infrastructure (gestion de l'énergie dans les bâtiments neufs et anciens), de gestion des déchets et des substances dangereuses, de gestion de l'eau, de déplacements et de politiques d'achats pour 2008-2010. En matière de gestion de l'énergie dans les bâtiments, tous les nouveaux projets de construction doivent répondre aux standards de haute performance énergétique. On en attend une réduction de la consommation d'énergie de $10 \%$ en moyenne. Les trois quarts des projets doivent être conçus en suivant la démarche haute qualité environnementale. Quelque 13000 bâtiments de la Défense dont la surface dépasse $1000 \mathrm{~m}^{2}$ sont équipés de compteurs individuels d'électricité, de chauffage et d'eau avant 2010. En matière de déchets et de substances dangereuses, l'objectif consiste à réduire la quantité de déchets et à améliorer leur recyclage par la définition d'un schéma directeur national d'implantation des stockages de matériels déclassés et de déchets, à généraliser les «passeports verts» aux navires en service (diminuer l'impact des substances dangereuses et faciliter le démantèlement) et à imposer aux industriels une cartographie des substances dangereuses dans les nouveaux programmes. En matière de transports, l'objectif national consiste à faire baisser de $20 \%$ les émissions de gaz à effet de serre avant 2020 (recours à la visioconférence et limitation des déplacements en voiture). 
Présenté comme une véritable stratégie de développement durable, ce nouveau plan vise à modifier l'approche de la Défense dans sa gestion des problématiques environnementales. Un nouveau bureau de niveau national est créé. Parallèlement, chaque armée ou grande direction du Ministère doit se doter d'un schéma directeur Environnement assorti d'objectifs planifiés. Ce plan représente un investissement de 180 millions d'euros. Chaque année, un bilan environnemental faisant état des consommations énergétiques et des rejets liquides et gazeux liés à l'activité de la Défense est établi. En 2008, quatre sites pilotes de la Défense font l'objet d'audits environnementaux complets : le parc d'hydrocarbures du Lazaret (Var), le camp de Mailly (Aube), la base aérienne 123 d’Orléans-Bricy (Loiret) et l’École polytechnique (Essonne).

En somme, dans la plupart des armées modernes occidentales, des stratégies de développement durable commencent à se faire jour. Une véritable prise de conscience de la notion de protection de l'environnement conduit à adopter des programmes ambitieux. Quels en sont les domaines d'application?

\section{Les domaines d'intervention des armées}

Les domaines d'intervention en matière de protection de l'environnement sont généralement divers. Comme le montrent les actions menées en France par le ministère de la Défense, ils s’inscrivent dans la gestion des sites militaires, l'encadrement des activités de défense et la dépollution des terrains militaires.

Le premier domaine concerne la protection des sites militaires. En France, cette orientation repose sur l'application du code de l'environnement, inspiré de la loi de mai 1930. En mai 1992, est adopté Natura 2000 qui vise à préserver la biodiversité sur le territoire de l'Union européenne en créant un réseau de sites naturels protégés (Montagut, 2003). Le programme est fondé sur une approche ouverte de la gestion des espaces tournés vers la conservation des habitats et la promotion des activités humaines.

En 2003, la participation de la Défense au réseau Natura 2000, dont le programme de classement est achevé en 2007, représente près de $20 \%$ de ses terrains, soit 50000 ha environ. Des camps de l'armée de terre ou des champs de tir font ainsi l'objet de mesures de classement et de protection en raison du caractère dit remarquable de la faune et de la flore, à l'écart des pollutions industrielles et de l'urbanisation croissante. Tel est le cas du camp de Bitche, en Moselle, utilisé pour le tir aux armes d'infanterie et constitué de zones arides et bourbeuses qui abritent quelques plantes rares. Une partie du camp de 182 ha est classé au titre de Natura 2000 sous la direction du commandant de la région et du parc naturel régional des Vosges. Il en est de même du camp de Montmorillon qui est l'objet d'une convention de gestion en 1997 entre la Circonscription militaire de défense de Limoges et le Conservatoire naturel régional de Poitou-Charentes. Utilisé de 1945 à 1966 pour l'entraînement de l'armée américaine et ensuite de l'armée française, ce camp de 1649 ha, constitué essentiellement de bocage et de landes, forme un élément typique du paysage du Montmorillonais. À l'écart de l'urbanisation et de l'agriculture intensive, il est désormais un terrain d'étude des botanistes et zoologistes tout en gardant sa vocation militaire. Dans le Cher, l'établissement technique de Bourges est un centre d'expertise de la Délégation générale pour l'armement (DGA) qui réalise des essais d'armes et de systèmes d'armes du combat 
aéroterrestre et naval (fusées d'artillerie notamment). Il comprend un champ de tir de $30 \mathrm{~km}$ de long englobant l'étang de Craon. Depuis 2003, le plan d'eau de 80 ha constitue une réserve ornithologique de première importance. La digue de l'étang est renforcée afin de préserver les prairies des inondations. Sur le site de Sedzère, au nord-est de Pau, occupé par la 13e Compagnie du 3e régiment du matériel, des mesures de restructuration sont appliquées au début des années $2000^{7}$. Les nouvelles constructions sont conçues pour éviter les risques de propagation d'explosions, tandis que les bâtiments de stockage sont réalisés «en igloo » afin de répondre aux normes de sécurité pyrotechniques internationales. Le stockage des munitions respecte des conditions de sécurité renforcées grâce à des équipements de surveillance supplémentaire et de nouveaux bacs de rétention. Un nouveau dépôt de munitions comprend un réseau de fossés reliés à des bassins d'orage dans le but de réguler les rejets d'eaux pluviales. Les eaux de lavage et les déchets sont recueillis dans une fosse étanche vidangée régulièrement par une société agréée. Conformément à la réglementation pour éviter tout risque de pollution des sols et des nappes phréatiques, les anciennes cuves à fioul et à carburants sont remplacées par des cuves à double paroi. Les déchets de tir sont triés suivant leur nature (acier, aluminium, bois, plastiques). Sur la zone de vie, différents conteneurs permettent de collecter le verre, les bouteilles de plastique, les papiers (journaux et revues), les ordures ménagères et enfin les déchets alimentaires. Chaque type de déchet fait l'objet de contrats d'enlèvement et de filières de traitement spécifiques à la nature des produits.

Le deuxième domaine d'intervention des armées porte sur l'encadrement des activités de défense. Dès 1810, des installations sont classées pour la protection de l'environnement, mais dans un but de protection de la santé publique. La loi de juillet 1976, puis le code de l'environnement s'inspirent de cette orientation et valorisent la notion d'environnement. Les installations sont répertoriées dans une nomenclature selon la gravité des dangers. L'exploitant est tenu au respect des normes et à l'obligation de remettre le site en état lors de la cessation de l'activité.

Selon les armes, des efforts sont faits pour réduire les effets nocifs sur l'environnement. Dans la marine, une démarche volontariste de respect de l'environnement est entreprise dès le début des années 1970 avec la prise de conscience de la pollution croissante de la mer et de la non-adaptation de l'architecture des bâtiments à la protection de l'environnement (Jaskierowicz et al., 2003). En 1984, les premières mesures restrictives aux rejets de déchets à la mer sont adoptées. En avril 1997, une directive prévoit le maintien des déchets non biodégradables à bord des bâtiments, la sensibilisation du personnel marin au tri sélectif et au stockage des déchets. Sur le porte-avions Charles-de-Gaulle, par exemple, une unité de traitement des déchets est créée ${ }^{8}$. Le porte-avions dispose ainsi de 10 jours d'autonomie de conservation

7 Depuis 1955, selon le ministère de la Défense, cette compagnie a pour mission principale le stockage, la surveillance technique et le ravitaillement des munitions d'instruction et d'intervention de l'ensemble des formations de la région Terre sud-ouest (RTSO). Les artificiers assurent également le désobusage des champs de tir, la destruction de munitions dangereuses ou déclassées, des enquêtes techniques et des études de sécurité.

8 Elle se compose de deux chaînes, la première, «liquide», concerne les déchets organiques. Des broyeurs transforment les aliments en pulpe et les jettent à la mer si le navire est au moins à 12 milles nautiques des côtes. La seconde chaîne, dite «solide», est employée pour les autres déchets. Elle traite de manière sélective les déchets et les déchiquète en fonction de leur nature (plastique, cellulose, métal, verre) pour les conserver en chambre froide jusqu'à leur récupération à terre ou par un autre navire. 
des déchets. À terre, les préoccupations environnementales sont du même ordre. Les ports militaires, intégrés à de grandes agglomérations comme Toulon (400 000 hab.) et Cherbourg (80 000 hab.), comprennent aussi des infrastructures à vocation nucléaire, pyrotechnique, des parcs à hydrocarbures dans des écosystèmes délicats.

La marine applique le protocole d'avril 1995 avec le ministère de l'Environnement. Des contrats sont conclus pour la qualité de l'eau et la préservation du milieu marin pour la baie de Brest (1998) et de Toulon (2002). Des opérations de revégétalisation et de gestion des zones boisées dans les emprises militaires sont organisées avec l'Office national des forêts. La marine procède également à des aliénations de sites remarquables au Conservatoire de l'espace littoral et des rivages lacustres. La convention de décembre 1994 prévoit la cession de 14 emprises dont 7 sont réalisées en 2003. Hors convention, 28 terrains de la marine sont cédés à différents acteurs territoriaux. La marine mène également des politiques diverses: usage des peintures antisalissures au tributylétain limité aux unités navigantes d'une surface de carène supérieure à $1500 \mathrm{~m}^{2}$, surveillance des polluants aériens et marins depuis 1994, gestion des déblais de dragage expérimentée dans le Var en 2003, réduction des rejets d'effluents dans l'atmosphère et dans l'eau ${ }^{9}$, limitation des nuisances sonores ${ }^{10}$.

La Marine dispose également de moyens de contrôle et de surveillance de l'environnement. Depuis 1997, le laboratoire d'analyses, de surveillance et d'expertise de la marine rassemble le laboratoire de surveillance radiologique et le laboratoire de chimie analytique. Il collabore avec des réseaux d'observation pour la surveillance du milieu marin, comme l'Ifremer (Institut français de recherche pour l'exploitation de la mer), et de la qualité des eaux des ports, notamment lors des grandes pollutions de l'Erika en 1999-2000 et du Prestige en 2002-2003. Dans le cadre de la prévention et de la lutte contre les pollutions, la marine, les affaires maritimes et la gendarmerie maritime disposent d'équipes d'intervention ${ }^{11}$.

La politique de protection de l'environnement par l'armée française s'oriente actuellement vers le remplacement de certaines substances et la fabrication de nouveaux types d'armes moins nocives. Dans le cadre du droit français et européen, des technologies anciennes du domaine matériaux sont interdites et remplacées. Le cadmium, utilisé pour le revêtement de protection des aciers contre la corrosion, est retiré, à l'exception de certaines dérogations dans l'aéronautique, en raison de sa toxicité (Veys et Gallicher, 2003). Des composés organiques de l'étain (oxydes ou fluorures de tributyl étain ou de triphényl étain), utilisés pour supprimer les salissures marines sur les coques immergées et dans les peintures marines antisalissures militaires, ont montré des actions mutagènes sur les mollusques comme les huîtres. Dès 1992, des

9 Le centre d'entraînement et de sécurité de Toulon est doté d'infrastructures de lavage des fumées et de filtrage des polluants.

10 Protocole du 14 février 2001 entre les ministères de la Défense et de l'Environnement pour réduire les nuisances. Il permet d'établir des chartes entre les bases aéronavales et les parties locales (associations, préfets, mairies).

11 Elles peuvent utiliser quatre avions de surveillance maritime de type Falcon 50 équipés d'un radar, d'un système infrarouge, de postes d'observation et de photographies. Un Falcon 50 est ainsi intervenu en Espagne dans le cadre de la pollution du Prestige. Un système de sémaphores permet d'obtenir une surveillance visuelle et radar continue le long des côtes. L'Institut de radioprotection et de sûreté nucléaire effectue des analyses de radioactivité dans la rade de Brest. 
mesures sont prises pour limiter l'emploi de ces produits. Elles sont renforcées en mai 2003 pour en éliminer toute utilisation avant 2007. En ce qui concerne la fabrication des munitions, de nouvelles technologies voient le jour. La Direction générale de l'Armement poursuit des recherches vers la mise au point de munitions "vertes » (Durliat, 2003). Déjà, dans plusieurs pays, sont adoptées des munitions de petit calibre (5,56 mm), comme en Suède et en Allemagne, ou des mines et grenades d'exercice biodégradables comme en France. Les recherches en cours jusqu'en 2008 tendent à remplacer le phosphore blanc par du phosphore rouge moins toxique, et à mettre au point une composition «verte» pour les contre-mesures pyrotechniques. D’autres recherches portent sur les "munitions à risques environnementaux atténués» et se concentrent sur les munitions de gros calibre (artillerie et char) et les contre-mesures pyrotechniques. Des études novatrices sont ainsi établies pour identifier les risques environnementaux des munitions en service ainsi que les solutions technologiques pour en limiter les effets.

Le troisième domaine d'intervention consiste à dépolluer les terrains. Cette orientation apparaît relativement récente. Selon les mesures antérieures à 2003, l'exploitant doit remettre en état le site tel qu'il ne s'y manifeste aucun danger. Sont pris en compte l'environnement, la commodité du voisinage, la santé, la sécurité et la salubrité publique, l'agriculture, la protection de la nature, la conservation des sites et des monuments. Mais l'application de ces directives conduit à des interprétations divergentes. La loi du 30 juillet 2003 relative à la prévention des risques techniques et naturels et à la réparation des dommages préserve ces orientations et les complète d'une nouveauté: le site doit permettre un usage futur déterminé conjointement entre l'exploitant ou le propriétaire et le maire ou le président de l'établissement de coopérative intercommunale. Le préfet peut également fixer des prescriptions plus contraignantes dans les documents d'urbanisme. La dépollution des terrains est devenue plus exigeante.

La dépollution du site de la poudrerie d’Angoulême, par le Service des programmes nucléaires et de missiles à partir de 2000, est un exemple significatif du respect de l'environnement (Levet et al., 2003). Dès le XIX ${ }^{e}$ siècle, la poudrerie d'Angoulême s'est spécialisée dans le domaine de la fabrication des produits pyrotechniques, voire la fabrication de toxiques de guerre, de 1914 à 1918. La surface des industries potentiellement polluantes s'étend à 100 ha en 2000 lors de la décision de la fermeture de la poudrerie. Le coton-poudre est le principal polluant, notamment en raison des risques d'explosion s'il se trouve à sec et en concentration suffisante dans le sol. Plusieurs dizaines de tonnes auraient été dispersées sur le site pendant un siècle d'utilisation intensive, y compris dans les réseaux souterrains reliant les différentes zones de fabrication. Le lac Vallez (1 ha), qui servait de décanteur pour les effluents des fabrications du site, contient des boues chargées de coton-poudre (4000 tonnes estimées). L'arrosage des berges par temps sec permet d'y maintenir des conditions de sécurité. Une zone sanctuarisée recouverte de terre abritait, jusqu'aux années 1970, des ateliers de coton-poudre. Elle présente à la fermeture du site une forte pollution liée à la fabrication des munitions et aux incidents ponctuels comme des fuites de canalisations qui ont eu pour conséquence de créer des cavités dans le sous-sol calcaire par l'infiltration des acides. D’autres pollutions peuvent être relevées en quantité moindre, comme la tolite et la nitroglycérine, les agents chimiques (ypérite, toxiques arsénisés) ou les restes du bombardement du 20 mars 1944 sur les usines (présence supposée de bombes incendiaires au phosphore non explosées enfouies dans le sol). 
Dès 2000, des opérations de dépollution sont entreprises. Un traitement thermique permet de détruire le coton-poudre du lac Vallez et devait s'achever en 2007. Les autres secteurs sont en cours d'études de dépollution. Les opérations se révèlent ainsi longues et coûteuses pour l'exploitant, mais au profit du respect de l'environnement et des populations avoisinantes.

En conséquence, dans la plupart des armées modernes, la prise de conscience de la protection de l'environnement devient une réalité concrète depuis les années 1990. De nouvelles réglementations sont appliquées parallèlement à des efforts volontaires de certaines armées. Elles concernent aussi bien la protection des espaces militaires que leur reconversion à des fins civiles. Les activités des armées sont également de plus en plus surveillées comme en témoignent les programmes de nouvelles munitions vertes.

\section{Environnement et culture militaire}

Alors que les stratégies militaires de développement durable commencent à se mettre en place dans les armées modernes, une culture militaire nouvelle tend à se dégager. Cette mutation culturelle semble suivre un mouvement planétaire en faveur de la protection de l'environnement. Ces stratégies sont-elles efficaces?

\section{Une révolution culturelle militaire}

Sans nul doute, la prise en compte de l'environnement dans les activités de défense constitue une révolution culturelle militaire, sinon un véritable tournant culturel. Après avoir été l'un des facteurs les plus forts de la destruction et de la dégradation du milieu naturel, ces armées consacrent désormais leurs efforts, en partie, à la protection de leurs sites militaires et à la réduction de leurs effets polluants.

$\mathrm{Au}$ sein de ces différents ministères de la défense (États-Unis, France, Grande-Bretagne entre autres), les efforts portent officiellement sur l'acquisition d'une culture de développement durable dans les processus de décision et sur les relations entre les directions militaires, les industriels et les autres ministères. Des réflexions en cours s'intéressent à la maîtrise des impacts environnementaux des systèmes d'armes et à l'approche intégrée des questions de santé, de sécurité et de protection de l'environnement. En France, la Délégation générale de l'armement tend à suivre ces engagements. Elle conçoit le développement durable en termes de cycle dans la réalisation des programmes d'armement comme la consommation des ressources en voie de raréfaction, le retrait et le recyclage des matériels et une démarche environnementale volontariste.

Les différents ministères de la défense concernés comprennent bien les avantages de telles politiques. Le thème de l'environnement devient un facteur de communication et de valorisation des activités de défense. Dans un contexte de mobilisation de l'opinion internationale contre le réchauffement climatique, ces ministères saisissent l'occasion de valoriser l'image de la défense. La stratégie de développement durable devient un vecteur de paix et de stabilité que les opinions publiques attendent et revendiquent. Dans un contexte de multiplication des opérations de maintien de la paix, depuis les années 1990, elle vient renforcer la dimension humanitaire des missions de reconstruction, qui nécessitent l'adhésion des populations locales. Elle participe à prévenir le «terrorisme environnemental», les conflits régionaux dont les conséquences sur l'environnement peuvent être catastrophiques. En somme, il y a une évolution de 
l’image donnée par les armées modernes, auparavant entachée par les interventions destructrices de l'environnement, comme au Viêtnam pour l'armée américaine entre 1965 et 1973, dont les effets sont encore dénoncés aujourd'hui.

Parallèlement à cet aspect, apparaît également un autre avantage en faveur autant de la défense que de la société civile. Les recherches technologiques menées par ces armées en matière de développement durable peuvent aussi connaître des débouchés dans le milieu civil. Cet aspect contribue à améliorer l'image de la défense tout en offrant un retour sur investissement diminuant le coût des programmes d'armement. En somme, les différentes politiques et stratégies de développement durable répondent à des objectifs avant tout politiques demandés par la société civile. En cela, elles représentent une rupture culturelle majeure au sein des armées, dont les missions étaient jusqu’alors fort éloignées de la protection de l'environnement.

\section{Des stratégies de développement durable sélectives}

Ces stratégies de développement durable demeurent toutefois partielles à l'échelle planétaire. Ce sont les armées modernes et occidentales qui engagent un tel processus. Or un certain nombre d'armées d’États émergents, dans les années 2000, sont en cours de restructuration et de redéveloppement. Les armées chinoises, russes et indiennes, pour ne citer que les plus importantes, renouvellent actuellement leurs forces et leurs équipements. Les préoccupations environnementales ne semblent pas être pour elles une priorité sociale et politique ni une priorité militaire.

Dans l'armée indienne, la sensibilisation aux questions environnementales reste limitée à la formation de l'élite des officiers de l'Académie nationale de défense, par des conférences et des projections de films, et à des actions éparses (D’Souza, 1995). Trois bataillons armés "écoterritoriaux» ont été créés, officiellement pour éviter la désertification au pied de l'Himalaya ${ }^{12}$. Des digues et des barrages régulateurs ont été construits. Sont aussi menées, auprès des populations locales, des campagnes de prévention de l'érosion du sol par l'utilisation de nouvelles sources d'énergie renouvelable (cuisinières solaires et installations au gaz à base de bouse de vache par exemple). Des mesures antipollution, notamment pour l'eau, et la plantation massive d'arbres (plus de 10 millions depuis 10 ans) sont également mises en œuvre. Toutefois, ces différentes actions restent isolées dans un contexte où la puissante armée indienne, en cours de réforme, se prépare à faire face à des menaces éventuelles de ses deux grands voisins (Pakistan et Chine). Si le cas indien affiche officiellement - malgré la rareté des études scientifiques sur ce sujet - une politique en faveur de l'environnement depuis la fin des années 1990, il ne semble pas exister une véritable stratégie de développement durable planifiée par la défense. Il en est de même, vraisemblablement, de toutes les autres armées, qu'elles soient russes ou chinoises, africaines ou sud-américaines.

En somme, à l'échelle mondiale, il semble bien que «le bon usage des armées pour l'environnement» soit encore à développer. Selon les informations disponibles, les actions menées par le pouvoir politique dans l'ensemble demeurent isolées lorsqu'elles existent. La fonction des armées reste encore liée essentiellement à l'action de coercition, comme l'a montré la présence de l'armée russe en Tchétchénie dans les années 1990 et 2000 ou, plus récemment, en Géorgie à l'été 2008.

12 Entre Dehra Dun et Mussoorie dans les collines Siwalik, dans les collines près de Jammu et le long du canal Ganga au Rajasthan. 


\title{
Quels résultats et suivis des stratégies de développement durable au sein des armées?
}

Quels sont les résultats et les suivis des politiques de développement durable au sein des armées? Compte tenu du caractère récent de la mise en place de ces politiques, les spécialistes ont relativement peu de recul sur les conséquences et les réalisations effectives. Jusqu'à présent, les principales informations proviennent des administrations concernées. Celles-ci ont tout intérêt à mettre en avant les programmes projetés ou les réalisations effectuées, à des fins de communication et de valorisation des activités de la Défense. Paradoxalement, tout au moins en France, l'opinion publique s'intéresse peu aux suivis de la Défense en la matière. Les critiques sont rares et les études universitaires inexistantes sur ce sujet.

Toutefois, il semble que ces politiques n’aboutissent pas forcément aux résutats escomptés. En Suisse, par exemple, les considérations environnementales au sein des armées sont pionnières parmi les armées occidentales. Le premier et dernier rapport environnemental de l'administration fédérale date de 2000. Selon Jean-Claude Renwald, membre du parti socialiste du canton du Jura et du Conseil national,

\begin{abstract}
ce rapport relevait à plusieurs reprises, pour le déplorer, l'absence de données quantitatives sur la pollution générée par l'armée suisse: "L’analyse environnementale a clairement montré que l'organisation actuelle de récolte et de gestion des données ne permet pas une évaluation quantitative exhaustive.» Plusieurs années plus tard, à l'exception de certains éléments livrés par le programme RUMBA (gestion des ressources et management environnemental de l'administration fédérale) des données précises en lien avec les activités de l'armée suisse ne sont toujours pas disponibles (2008).
\end{abstract}

Si des mesures contre la pollution par le matériel militaire (véhicules, avions, etc.) sont prises, on déconce, en mars 2008, l'absence de résultats sur les impacts de leur application plusieurs années après.

Au final, en Suisse comme dans d'autres pays, les impacts sur l'environnement, de ces stratégies de développement durable sont peu connus. En France, il semble que les seules informations chiffrées, en 2009, portent sur la répression des atteintes à l'environnement qui représente 70000 contraventions ou délits par an. En 2002, la gendarmerie nationale en a constaté plus de 40000 dont 6000 délits. À partir de ce constat, deux conclusions s'imposent. La première permet d'observer l'importance médiatique des effets d'annonce de la mise en place de ces politiques sans qu'une administration spécialisée suive les résultats des procédures adoptées. Pour répondre à cette demande d'information, le ministère de la Défense français a créé, en 2008, un bureau comprenant huit personnes coordonnant l'action de 300 chargés de protection de l'environnement pour réaliser le bilan environnemental annuel. En 2010, force est de remarquer qu'aucun rapport officiel et public n'a encore été publié sur ce sujet. Les derniers efforts du plan 2008-2010 demeurent expérimentaux, comme en témoigne le choix des quatre sites pilotes pour réaliser des audits d'environnement externes. Quant aux autres pays, bien que l'état de la recherche doive être approfondi, la même déficience d'informations subsiste. À la demande d'un écobilan de l'armée suisse du député d'opposition Renwald, en 2008, le Conseil fédéral lui répond qu'il n’apporterait pas de plus-value significative en rapport avec les informations et les prestations en matière d'environnement. La seconde conclusion met aussi en évidence le caractère sensible de ce type d'information. À l'aube d'une prise de conscience du rôle des 
armées en matière d'environnement, les moyens comme l'application des premières mesures - restent, semble-t-il, encore limités. Dans le contexte de l'après conférence de Copenhague de décembre 2009, qui a révélé les tensions entre les États sur une politique commune contre le réchauffement climatique, la démarche environnementale par les armées apparaît encore restreinte et, peut-être, difficilement compatible avec l'essence même de l'activité militaire, d'abord coercitive, elle-même fortement consommatrice d'énergies et fortement polluante.

\section{Conclusion}

L'environnement constitue un élément essentiel pour le militaire en opération. Selon la nature de la végétation, ses actions se déploient très différemment. Depuis les origines de la guerre, cet environnement est considéré à la fois comme une arme de guerre et une source croissante de tensions. Toutefois, depuis les bombardements répétés de l'armée américaine durant la guerre du Viêtnam, se rencontre une nouvelle tendance qui montre une prise de conscience des armées occidentales en faveur de l'environnement. Sur les théâtres d'opérations extérieures, comme en ex-Yougoslavie, au Rwanda, au Soudan ou en Irak, la gestion des ressources naturelles et de l'écosystème devient une préoccupation parmi d'autres des militaires, car elle favorise les sorties de crise. L'établissement d'un corpus de règles internationales est une des réalisations, interdisant, par exemple, l'utilisation de l'eau comme arme de guerre. Il témoigne d'un changement des mentalités collectives, y compris dans un domaine qui faisait de l'environnement une des victimes principales de tout conflit. Aujourd'hui, la coopération entre Défense et Environnement dans les États développés devient de plus en plus une nécessité. La gestion, le traitement et le contrôle des données environnementales sont considérés comme un enjeu majeur pour les générations futures, comme le souligne le récent plan environnemental de 2008 en France. L'action du militaire se montre ainsi écologique en participant à la protection de la nature et à la préservation de l'écosystème. Il en résulte une révolution culturelle militaire certaine, mais celle-ci apparaît, malgré tout, limitée à quelques armées modernes dont les résultats sont à étudier à long terme. Le «bon usage de l'environnement par les armées» reste, en grande partie dans le monde, lié aux multiples sources de tension et à son exploitation à des fins coercitives. 


\section{Bibliographie}

BOULANGER, Philippe (2009) Géographie et culture militaire française $\left(X X^{e}-X X I^{e}\right.$ siècles). Mémoire inédit d'habilitation à diriger des recherches.

BOULANGER, Philippe (2006) Géographie militaire. Paris, Ellipses.

CHAIX, Nicole (dir.) (2006) Armement et désordre mondial. Association des auditeurs du Chear.

D'SOUZA, Eustache (1995) Rôle potentiel de l'armée dans la protection de l'environnement: le cas de l'Inde. Unasylva, 183. [En ligne.] http://www.fao.org/docrep/ v7850F/v7850f12.htm

DURSCHMIED, Erik (2004) Le facteur climat, quand les forces de la nature ont changé le cours de l'histoire. JC Lattès-Trinacra.

DURIAT, Emmanuel (2003) Les études amont pour munitions «vertes». L'Armement, n84, p. 101-104.

GANDUBERT, Claudie (2003) Le droit de l'environnement et les activités de défense. L'Armement, n 84, p. 29-34.

GUÉVEL, Philippe (2003) USA Greening the government through leadership in environmental management, la DOD se met au vert. L'Armement, n84, p. 144-147.

JASKIEROWICZ, Donald et al. (2003) La Marine nationale et l'environnement. L'Armement, n84, p. 52-60.

LACOSTE, Yves (2008) La géographie, la géopolitique et le raisonnement géographique, Hérodote, $\mathrm{n}^{\circ} 130$, p. 17-42, et p. 30-31.

LEVET, Raymond, PECHOUX, Frédéric, PEZRON, Christophe et LIÉGEOIS, Pascal (2003) Dépollution des anciens sites d'armement: pilotage et aspects techniques. L'Armement, n84, p. 137-143.

MARIONNET, Joël (2003) La prise en compte du risque chez les militaires en opération. L'Armement, n84, p. 41-44.
MINISTÈRE DE LA DÉFENSE (1980) Notice sur les actions du génie en campagne, participation à la contre-mobilité. Direction centrale du génie, GEN 213.

MOLLARD-BANNELIER, Karine (2001) La protection de l'environnement en temps de conflit armé. Paris, A. Padone.

MONTAGUT, Géraud (2003) La défense et Natura 2000. L'Armement, n ${ }^{\circ}$ ', p. 51.

PEARCE, Fred (2000) Guerres et environnement: réaction en chaîne, Regard européen, août, p. 120-122.

RENWALD, Jean-Claude (2008) Pour un écobilan de l'armée suisse. Objets parlementaires. The Federal Assembly - The Swiss Parliament. [En ligne.] http://www. parlament.ch/E/Suche/Pages/geschaefte. aspx?gesch_id=20083025.

SCHMITZ, Marc, AWOONOR, Kofi, COUSTEAU, Jacques-yves et VAN YPERSELE, Jean-Pascal (1999) Les conflits verts, la dégradation de l'environnement source de tensions majeures. GRIP, $\mathrm{n}^{\circ} 22$.

SIRONNEAU, Jacques (2003) Guerre et environnement. Dans François Daguzan et Pascal Lorot (dir.) Guerre et économie. Ellipses, p. 91-113

SUN, Tse (1993) L'art de la guerre, Paris, Pocket.

VADROT, Claude-Marie (2005) Guerres et environnement, Panorama des paysages et des écosystèmes bouleversés. Paris, Delachaux et Niestlé.

VEYRET, Yvette (1997) Géo-environnement. Paris, Sedes.

VEYS, Jean-Marie et GALLICHER, Geneviève (2003) Matériaux pour la Défense et respect de l'environnement. L'Armement, $n^{\circ} 84$, p. 82-86. 